Rev. Biol. Trop., 48(2/3): 665-670, 2000

www.ucr.ac.cr www.ots.ac.cr www.ots.duke.edu

\title{
Nueva especie de Anolis (Sauria: Iguanidae) de la región norte de Cuba oriental
}

\author{
Ansel Fong G.' y Orlando H. Garrido² \\ 1 BIOECO, Museo de Historia Natural “T. Romay”, Enramadas \# 601, Santiago de Cuba 90100, Cuba; ansel@bioe- \\ co.ciges.inf.cu \\ 2 Museo Nacional de Historia Natural, Obispo \# 61, Plaza de Armas, Habana Vieja, Cuba.
}

Recibido 10-VI-1999. Corregido 27-I-2000. Aceptado 4-II-2000.

\begin{abstract}
A new Anolis species of the Alpha section from the north region of eastern Cuba (Holguín province) is described. It differs from all Cuban species of Anolis in its green coloration with greenish gray bands on body, legs and tail, in having subtriangular mental scales as well as in other details of color and scutellation. This new species is most closely related to $A$. isolepis but it can be distinguished from both, $A$. $i$. isolepis and A. i. altitudinalis, by its coloration and pattern, the larger body size, the presence of smooth ventral scales (similar in size to the dorsal scales) and by the absence of enlarged postcloacal scales in the male.
\end{abstract}

Key words: Sauria, Iguanidae, Anolis, new species, Cuba.

El eminente herpetólogo recién fallecido Ernest E. Williams expuso en uno de sus trabajos "la era de los descubrimientos no ha terminado" (Williams 1976), postulado que se hace vigente si analizamos el número de especies de Anolis (incluido Chamaeleolis) registradas para Cuba en fechas sucesivas: 23 especies por Ruibal (1964), 44 especies por Garrido y Jaume (1984) y 53 especies por Powell et al. (1996), y más teniendo en cuenta que después de esta fecha un nuevo taxon ha sido publicado y otros dos están en prensa. Dos factores básicos han influido notablemente en este incremento: el mayor interés por el grupo y el gran número de expediciones a regiones poco o nada conocidas herpetológicamente.

Durante una de las expediciones de monitoreo de biodiversidad a la altiplanicie del Toldo, Holguín, el entomólogo Luis F. de Armas capturó un macho adulto de una especie nueva del género Anolis, la cual describimos en el presente trabajo.
Williams (1976) dividió las lagartijas del género Anolis en dos grandes secciones, Alpha y Beta. Ambas secciones están representadas en Cuba, estando incluidas en la sección Alpha todas las especies de coloración verde: A. porcatus, A. allisoni, A. isolepis, A. cyanopleurus y el complejo Anolis equestris. La especie que aquí describimos comparte su coloración con estos táxones y de acuerdo a sus características quedaría incluida en el grupo, subgrupo y superespecie carolinensis.

\section{MATERIALES Y MÉTODOS}

Los caracteres morfométricos y merísticos considerados fueron los clásicos empleados en las especies cubanas del género Anolis (ver Garrido y Estrada 1989, Estrada y Garrido 1991). Las escamas dorsales, ventrales y gulares se contaron en una distancia igual a aquella entre el ángulo anterior de la órbita y la punta 
del hocico. Además se midió la longitud máxima y el ancho máximo de las escamas mentales y se calculó el cociente longitud/ancho.

Todas las medidas se tomaron en milímetros con pie de rey o con ocular micrométrico bajo el microscopio estereoscópico, en ambos casos con exactitud de $0.05 \mathrm{~mm}$. Para las mediciones se muestra el promedio seguido de los valores mínimos y máximos entre paréntesis. Las colecciones se identifican con los siguientes acrónimos: colección herpetológica de BIOECO, Santiago de Cuba=BSC.H; Museo de Historia Natural "Carlos de la Torre", Holguín=MHNH; Instituto de Ecología y Sistemática, La Habana $=$ CZACC.

\section{SISTEMATICA}

\section{Anolis toldo, especie nueva}

Holótipo: Macho adulto BSC.H-1080, recolectado en la altiplanicie del Toldo $(800 \mathrm{~m}$ de altitud), Moa, provincia Holguín, Cuba, el 19 de junio de 1997 por Luis F. de Armas.

Diagnosis: La coloración verde de A. toldo la distingue de la mayoría de las especies del género en Cuba, por lo que sólo se comparará con aquellas especies que presentan dicha coloración. El complejo Anolis equestris está formado por las especies gigantes de Cuba, diferenciándose fácilmente de $A$. toldo por presentar gran tamaño y las escamas de la cabeza osificadas. Por el contrario, A. cyanopleurus es una especie pequeña, alargada, con grandes escamas dorsales y ventrales aquilladas y con la cola 2.5 veces más larga que el cuerpo, lo cual la diferencia de $A$. toldo, que presenta escamas ventrales lisas y la cola sólo ligeramente más larga que el cuerpo.

Las especies Anolis allisoni y A. porcatus tienen gránulos en el dorso y crestas cantales bien desarrolladas, pero el patrón de franjas verticales oscuras y el tono del verde de $\mathrm{A}$. tol$d o$ es diferente al de estas dos especies, que se diferencian además por el color azul y verde de los machos de A. allisoni y la presencia de una marcada banda longitudinal mediodorsal clara en hembras y en los machos de poblaciones

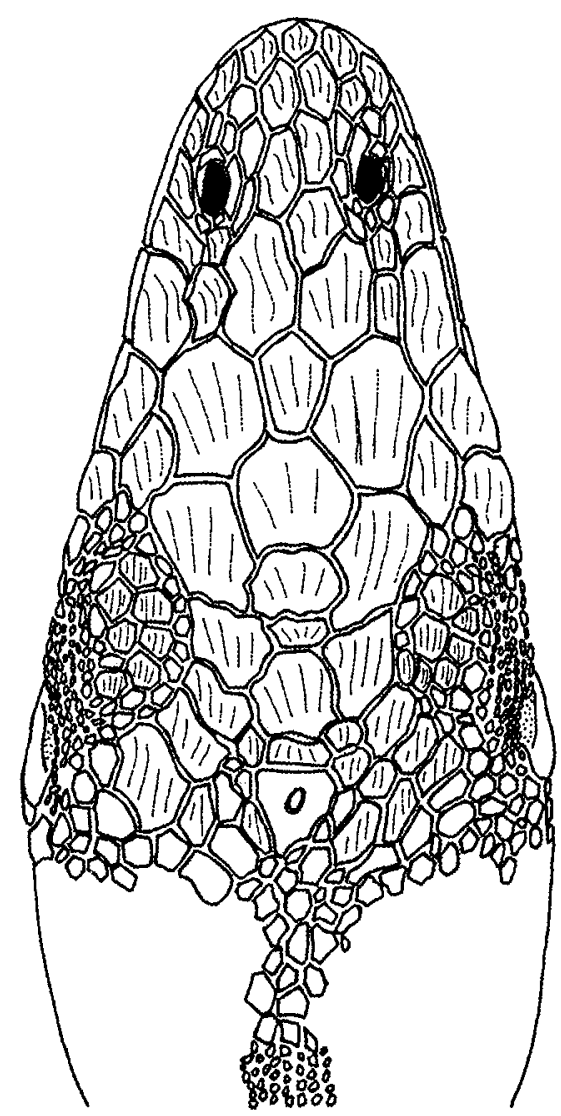

Fig. 1. Vista dorsal de la cabeza de Anolis toldo (holótipo, BSC.H-1080).

Fig. 1. Dorsal surface of the head of Anolis toldo (holotype, BSC.H-1080).

orientales de A. porcatus. Otras características diferenciales son el oído alargado y el pliegue gular de color malva en Anolis allisoni y de color rosado en A. porcatus. En A. toldo el oído es redondeado y el pliegue gular amarillo claro.

La especie con la cual presenta mayor similitud es con A. isolepis, con la que se realizará la comparación más detallada. Se analizarán por separado A. isolepis isolepis y A. isolepis altitudinalis porque es muy probable que esta última sea en realidad una especie del complejo y no una raza geográfica. Una revisión futura del grupo debe aclarar la situación.

De la subespecie isolepis se distingue por un mayor tamaño, $61.2 \mathrm{~mm}$ vs. $42 \mathrm{~mm}$ de 
longitud hocico-cloaca; coloración verde clara uniforme en $A$. isolepis, pudiendo adquirir tonalidades violáceas con reticulaciones oscuras pero nunca con las franjas verticales gris-verdosas oscuras bien marcadas y constantes en $A$. toldo; mayor tamaño del fémur y mayor cociente femoral, 13.2 y 4.6 vs. $8.5(7.2-9.3)$ y 4.3 (3.4-4.8); mayor longitud y ancho de la cabeza, 19.2 y 10.5 vs. $12.6(11.5-13.1)$ y $6.7(5.8-7.2)$; mayor cociente de la cabeza, 3.2 vs. 2.9 (2.43.1 ); las escamas ventrales tienen aproximadamente el mismo tamaño que las dorsales, por lo que su número es similar, 30 , a diferencia de isolepis que posee las ventrales de mayor tamaño que las dorsales, por lo que hay menor número de ventrales, 24.4 (21-28), que dorsales, 30 (25-33); mayor número de escamas gulares, 24 vs. 19.2 (18-21); mayor número de infralabiales, 10 vs. $7-9($ moda $=7)$; las escamas superciliares agrandadas son de menor tamaño y por tanto hay un mayor número en $A$. toldo, 13 , que en isolepis, 7; la forma y tamaño de las escamas mentales es diferente (Fig. 2), siendo de mayor tamaño en A. toldo, 1.7 por $1.4 \mathrm{~mm}$ vs. 1.5 por $0.8 \mathrm{~mm}$, con un menor cociente, 1.2 vs. 1.9 (1.5-2.1). Las escamas ventrales son totalmente lisas, mientras que en isolepis pueden ser levemente o muy aquilladas, pero no totalmente lisas. En la región del pecho y la base del pliegue gular las escamas están muy separadas unas de otras, con espacios de piel entre ellas, al contrario de isolepis que presenta estas escamas bien unidas y sin espacios de piel entre ellas; no posee escamas postcloacales agrandadas, las que son bien conspicuas en los machos de isolepis; presenta una cresta frontal desarrollada, siendo las escamas de la cabeza planas y en forma de pavimento en isolepis.

De A. isolepis altitudinalis se diferencia por las mismas características de coloración que isolepis isolepis; por el mayor tamaño, 61.2 $\mathrm{mm}$ vs. $52 \mathrm{~mm}$ de longitud hocico-cloaca; menor cociente femoral, 4.6 vs. 5.3 (5.0-5.8); mayor cociente de la cabeza, 3.2 vs. 2.7 (2.6-2.8); menor número de escamas dorsales 30 vs. 36 ; menor número de escamas ventrales 30 vs. 38.0 (32-45); menor número de infralabiales 10 vs. 11; mayor número de escamas superciliares agrandadas, 13 vs. $5-6$; menor número de escamas entre los primeros cantales, 5 vs. 5-7 (mo$\mathrm{da}=7$ ); escamas ventrales lisas, muy aquilladas en altitudinalis; escamas del pecho y la base del pliegue gular separadas, bien unidas en altitudinalis (igual que en isolepis), sin escamas postcloacales agrandadas, bien notables y agrandadas en los machos de altitudinalis.

Descripción del holótipo: Macho adulto, $61.2 \mathrm{~mm}$ de longitud hocico-cloaca, cabeza con $19.2 \mathrm{~mm}$ de longitud y $10.5 \mathrm{~mm}$ de ancho, 3.2 de cociente; escamas del hocico aquilladas; cinco escamas postrostrales; cinco escamas entre los primeros cantales; una fila de escamas entre los semicírculos supraorbitales; $1 / 1$ escamas entre el interparietal y los semicírculos; interparietal más larga que ancha, $1.1 \mathrm{~mm}$ de largo por $0.9 \mathrm{~mm}$ de ancho; 13 escamas superciliares
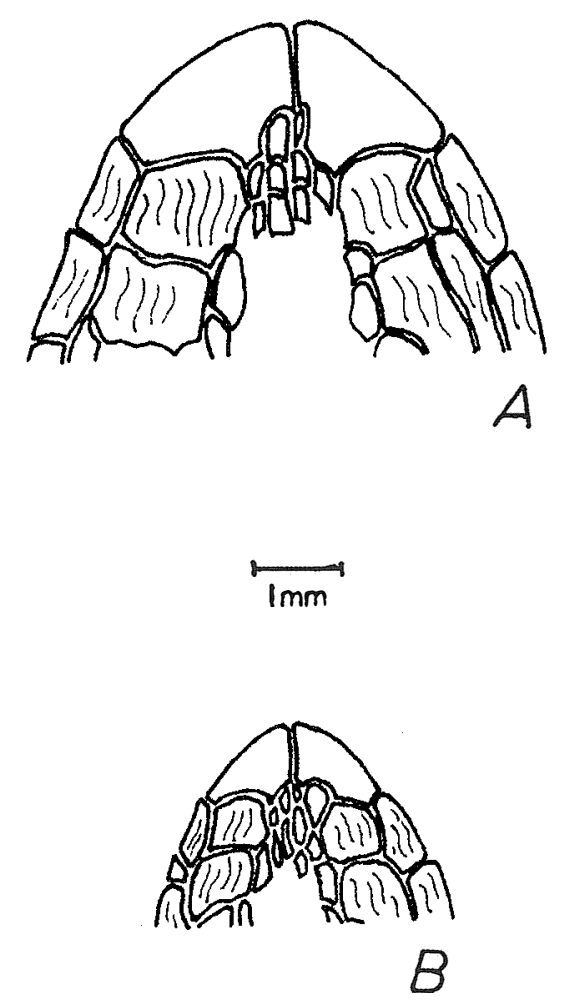

Fig. 2. Escamas de la región mental de (A) Anolis toldo (holótipo, BSC.H-1080), (B) A. isolepis isolepis (BSC.H-1695).

Fig. 2. Chin scalation of (A) Anolis toldo (holotype, BSC.H-1080), (B) A. isolepis isolepis (BSC.H-1695). 
agrandadas, algunas sin agrandar; nueve escamas supralabiales; 10 escamas infralabiales; 17 escamas loreales; escamas mentales subtriangulares, $1.7 \mathrm{~mm}$ de largo por $1.4 \mathrm{~mm}$ de ancho; cuatro escamas postmentales; 24 escamas gulares; abertura auditiva redondeada, aproximadamente un milímetro de diámetro; 30 escamas dorsales; 30 escamas ventrales lisas y similares en tamaño a las dorsales; sin escamas postcloacales agrandadas. Pliegue gular de mediano tamaño, con las escamas interiores muy separadas en su base y con espacios de piel entre ellas. Fémur con una longitud de $13.2 \mathrm{~mm}$; un cociente femoral de 4.6; 22 laminillas bajo el 4to dedo de la mano derecha; escamas de los supracarpos y supratarsos multicarinadas; escamas sobre el fémur lisas; cola sin cresta, cilíndrica y más larga que el cuerpo, con una longitud de $71.4 \mathrm{~mm}$; con verticilios evidentes.

Coloración en vida: Color general verde, un tanto oliváceo, con cinco franjas verticales de color gris verdoso entre las extremidades anteriores y posteriores. Una serie de franjas verticales más finas, también de color gris verdoso, en el cuello, las extremidades y la cola. Hocico de color verde oscuro con ocelos de color gris-verdoso. Franja supralabial amarilla clara extendiéndose hasta detrás de las extremidades anteriores. Iris negro. Carpos, tarsos y dedos de color pardusco con puntos grises. Vientre jaspeado en pardo y verde claro, garganta con bandas longitudinales pardas sobre fondo verdoso. Pliegue gular amarillo claro con las escamas interiores blancas. En fase oscura toma una coloración parda, casi negra, las extremidades se tornan verde-olivo oscuro y las franjas verticales de todo el cuerpo se oscurecen aun más.

Coloración en alcohol: Cuello, extremidades y cola verde oscuro con un ligero tinte grisáceo, el resto pardo claro. Todas las franjas del cuerpo de color pardo oscuro. Las manchas amarillentas del hocico poco notables. Franja supralabial y pliegue gular con una coloración amarillenta, pero muy clara. Vientre y garganta beige con las marcas de color pardo. Carpos, tarsos y dedos de color pardo, no se observan los puntos grises presentes en vida.
Historia natural: El ejemplar se capturó en la rama de un arbusto a una altura de $1.40 \mathrm{~m}$ sobre el suelo, a aproximadamente las 2: 00 p.m. El arbusto se encontraba en un área de vegetación aislada en el centro de un camino, la cual ocupaba un área de unos $15 \times 3 \mathrm{~m}$. El tipo de vegetación donde se colectó $A$. toldo pertenece a una pluvisilva esclerofila montana baja sobre serpentina (Capote 1997) y se caracteriza por poseer emergentes de 10 a $12 \mathrm{~m}$ de altura, un estrato arbóreo denso de 4 a $6 \mathrm{~m}$, un estrato arbustivo de $2 \mathrm{~m}$ y un estrato herbáceo ralo de $70 \mathrm{~cm}$ de altura. El ejemplar en vida producía chillidos similares a los de A. isolepis y trataba de morder constantemente. Simpátricamente con A. toldo vive A. isolepis isolepis.

Distribución: Conocido solamente de la altiplanicie del Toldo, Moa, provincia Holguín, Cuba. Este sistema geográfico pertenece a las Alturas de Moa y se encuentra situado entre el río Moa por el oeste y el río Jiguaní por el este, a lo largo de unos $16 \mathrm{~km}$. El rango altitudinal de la altiplanicie oscila entre 600 y $900 \mathrm{~m}$ s.n.m., con la altitud máxima de $1175 \mathrm{~m}$ en el Pico El Toldo.

Etimología: En alusión a la localidad tipo, la altiplanicie del Toldo.

Material examinado: Anolis isolepis isolepis.- Provincia Holguín: Altiplanicie del Toldo, Moa (BSC.H-1032 y 1986); Arroyo Bueno, La Melba, Moa (BSC.H-1240); Mina Merceditas, Moa (BSC.H-1238); Río Jaguaní, camino Yarey-Merceditas (BSC.H-1315); Meseta Pinares de Mayarí, Mayarí (MHNH 14-260); Cabonico, Mayarí (MHNH 14-265); La Zoilita, Sierra del Cristal, Mayarí (MHNH 14-263); Playa Pesquero, R. Freyre (MHNH 14-262); El Peñón, Estero Ciego, R. Freyre (MHNH 14-279); El Jobal (Los Hoyos), Gibara (MHNH 14-278). Provincia Santiago de Cuba: La Gran Piedra (BSC.H-1843). Provincia Guantánamo: Sabana, Maisí (BSC.H-1695); Camino a Santa Rosa, Sabana, Maisí (MHNH-sin número [Número original NNP-313]); Campamento Ruso, Yateras (MHNH 14-261). Sin datos (MHNH 14-264).

Anolis isolepis altitudinalis.- Provincia Santiago de Cuba: Alto del Cardero (1050 m s.n.m.), Pico Turquino, Sierra Maestra 
(CZACC 4-7027, holótipo y otros cuatro ejemplares, parátipos, sin número).

\section{AGRADECIMIENTOS}

A Luis F. de Armas, quien gentilmente nos donó el ejemplar recolectado por él, hasta ahora el único conocido de esta especie; a todos los participantes en el monitoreo de biodiversidad de la altiplanicie del Toldo por su apoyo y ayuda en el trabajo de campo, a Nils Navarro por el préstamo del material bajo su cuidado y por sus sugerencias durante las primeras etapas de realización del trabajo y a dos revisores anónimos por sus útiles comentarios.

\section{RESUMEN}

Se describe una nueva especie de Anolis de la sección Alpha para la región norte de Cuba oriental (provincia Holguín). Difiere del resto de las especies cubanas del género en su color verde con franjas verticales gris verdosas en el cuerpo, la cola y las extremidades, en la forma subtriangular de las escamas mentales y en otras características de coloración y escamación. La especie más parecida es $A$. isolepis, aunque varios caracteres diferencian a sus dos subespecies de la nueva especie descrita.

\section{REFERENCIAS}

Capote, R. P. 1997. Informe parcial del monitoreo florístico al proyecto de exploración orientativa Piloto. Cesigma División América, La Habana. 82 p.

Estrada, A. R. \& O. H. Garrido. 1991. Dos nuevas especies de Anolis (Lacertilia: Iguanidae) de la Región Oriental de Cuba. Carib. J. Sci. 27: 146-161.

Garrido, O. H. \& A. R. Estrada. 1989. Nueva especie del complejo Anolis alutaceus (Lacertilia: Iguanidae) para Cuba. Rev. Biol. 3: 57-66.

Garrido, O. H. \& M. L. Jaume. 1984. Catálogo descriptivo de los anfibios y reptiles de Cuba. Doñana, Acta Vertebrata 11: 5-128.

Powell, R., R. W. Henderson, K. Adler, \& H. A. Dundee. 1996. An annotated checklist of West Indian Amphibians and Reptiles, p. 51-93. In R. Powell y R. W. Henderson (eds.). Contributions to West Indian Herpetology: A tribute to Albert Schwartz. SSAR. Ithaca, Nueva York.

Ruibal, R. 1964. An annotated checklist and key to the anoline lizards of Cuba. Bull. Mus. Comp. Zool. 130: 476-520.

Williams, E. E. 1976. West Indian anoles: a taxonomic and evolutionary summary. I. Introduction and a species list. Breviora 440: 1-21. 